\title{
WEIGHTED ESTIMATES FOR BELTRAMI EQUATIONS
}

\author{
Albert Clop and Victor Cruz \\ Universitat Autònoma de Barcelona, Departament de Matemàtiques \\ 08193 Bellaterra, Barcelona, Catalonia; albertcp@mat.uab.cat \\ Universidad Tecnológica de la Mixteca, Instituto de Física y Matemáticas \\ 69000 Huajauapan de León, Oaxaca, México; victorcruz@mixteco.utm.mx
}

\begin{abstract}
We obtain a priori estimates in $L^{p}(\omega)$ for the generalized Beltrami equation, provided that the coefficients are compactly supported $V M O$ functions with the expected ellipticity condition, and the weight $\omega$ lies in the Muckenhoupt class $A_{p}$. As an application, we obtain improved regularity for the jacobian of certain quasiconformal mappings.
\end{abstract}

\section{Introduction}

In this paper, we consider the inhomogeneous, Beltrami equation

$$
\bar{\partial} f(z)-\mu(z) \partial f(z)-\nu(z) \overline{\partial f(z)}=g(z), \quad \text { a.e. } z \in \mathbf{C},
$$

where $\mu, \nu$ are $L^{\infty}(\mathbf{C} ; \mathbf{C})$ functions such that $\||\mu|+|\nu|\|_{\infty} \leq k<1$, and $g$ is a measurable, $\mathbf{C}$-valued function. The derivatives $\partial f, \bar{\partial} f$ are understood in the distributional sense. In the work [3], the $L^{p}$ theory of such equation was developed. More precisely, it was shown that if $1+k<p<1+\frac{1}{k}$ and $g \in L^{p}(\mathbf{C})$ then (1) has a solution $f$, unique modulo additive constants, whose differential $D f$ belongs to $L^{p}(\mathbf{C})$, and furthermore, the estimate

$$
\|D f\|_{L^{p}(\mathbf{C})} \leq C\|g\|_{L^{p}(\mathbf{C})}
$$

holds for some constant $C=C(k, p)>0$. For other values of $p,(1)$ the claim may fail in general. However, in the previous work [9], Iwaniec proved that if $\mu \in V M O(\mathbf{C})$, then for any $1<p<\infty$ and any $g \in L^{p}(\mathbf{C})$ one can find exactly one solution $f$ to the $\mathbf{C}$-linear equation

$$
\bar{\partial} f(z)-\mu(z) \partial f(z)=g(z)
$$

with $D f \in L^{p}(\mathbf{C})$. In particular, (2) holds whenever $p \in(1, \infty)$. Recently, Koski [11] has extended this result to the generalized equation (1). For results in other spaces of functions, see [5].

In this paper, we deal with weighted spaces, and so we assume $g \in L^{p}(\omega), 1<$ $p<\infty$. Here $\omega$ is a measurable function, and $\omega>0$ at almost every point. By checking the particular case $\mu=\nu=0$, one sees that, for a weighted version of the estimate (2) to hold, the Muckenhoupt condition $\omega \in A_{p}$ is necessary. It turns out that, for compactly supported $\mu \in V M O$, this condition is also sufficient.

Theorem 1. Let $1<p<\infty$. Let $\mu$ be a compactly supported function in $V M O(\mathbf{C})$, such that $\|\mu\|_{\infty}<1$, and let $\omega \in A_{p}$. Then, the equation

$$
\bar{\partial} f(z)-\mu(z) \partial f(z)=g(z)
$$

doi:10.5186/aasfm.2013.3818

2010 Mathematics Subject Classification: Primary 30C62, 35J15, 42 B37.

Key words: Beltrami equation, quasiconformal mapping, Muckenhoupt weight. 
has, for $g \in L^{p}(\omega)$, a solution $f$ with $D f \in L^{p}(\omega)$, which is unique up to an additive constant. Moreover, one has

$$
\|D f\|_{L^{p}(\omega)} \leq C\|g\|_{L^{p}(\omega)}
$$

for some $C>0$ depending on $\mu, p$ and $[\omega]_{A_{p}}$.

The proof copies the scheme of [9]. In particular, our main tool is the following compactness Theorem, which extends a classical result of Uchiyama [18] about commutators of Calderón-Zygmund singular integral operators and VMO functions.

Theorem 2. Let $T$ be a Calderón-Zygmund singular integral operator. Let $\omega \in$ $A_{p}$ with $1<p<\infty$, and let $b \in V M O\left(\mathbf{R}^{n}\right)$. The commutator $[b, T]: L^{p}(\omega) \rightarrow L^{p}(\omega)$ is compact.

Theorem 2 is obtained from a sufficient condition for compactness in $L^{p}(\omega)$. When $\omega=1$, this sufficient condition reduces to the classical Frechet-Kolmogorov compactness criterion. Theorem 1 is then obtained from Theorem 2 by letting $T$ be the Beurling-Ahlfors singular integral operator.

A counterpart to Theorem 1 for the generalized Beltrami equation,

$$
\bar{\partial} f(z)-\mu(z) \partial f(z)-\nu(z) \overline{\partial f(z)}=g(z),
$$

can also be obtained under the ellipticity condition $\||\mu|+|\nu|\|_{\infty} \leq k<1$ and the $V M O$ smoothness of the coefficients (see Theorem 8 below). Theorem 2 is again the main ingredient. However, for (3) the argument in Theorem 1 needs to be modified, because the involved operators are not $\mathbf{C}$-linear, but only $\mathbf{R}$-linear. In other words, C-linearity is not essential. See also [11].

It turns out that any linear, elliptic, divergence type equation can be reduced to equation (3) (see e.g. [2, Theorem 16.1.6]). Therefore the following result is no surprise.

Corollary 3. Let $K \geq 1$. Let $A: \mathbf{R}^{2} \rightarrow \mathbf{R}^{2 \times 2}$ be a matrix-valued function, satisfying the ellipticity condition

$$
\frac{1}{K} \leq v^{t} A(z) v \leq K, \quad \text { whenever } v \in \mathbf{R}^{2},|v|=1,
$$

at almost every point $z \in \mathbf{R}^{2}$, and such that $A-\mathbf{I d}$ has compactly supported $V M O$ entries. Let $p \in(1, \infty)$ be fixed, and $\omega \in A_{p}$. For any $g \in L^{p}(\omega)$, the equation

$$
\operatorname{div}(A(z) \nabla u)=\operatorname{div}(g)
$$

has a solution $u$ with $\nabla u \in L^{p}(\omega)$, unique up to an additive constant, and such that

$$
\|\nabla u\|_{L^{p}(\omega)} \leq C\|g\|_{L^{p}(\omega)}
$$

for some constant $C=C(A, \omega, p)$.

Other applications of Theorem 1 are found in connection to planar $K$-quasiconformal mappings. Remember that a $W_{\text {loc }}^{1,2}$ homeomorphism $\phi: \Omega \rightarrow \Omega^{\prime}$ between domains $\Omega, \Omega^{\prime} \subset \mathbf{C}$ is called $K$-quasiconformal if

$$
|\bar{\partial} \phi(z)| \leq \frac{K-1}{K+1}|\partial \phi(z)| \text { for a.e. } z \in \Omega .
$$

In general, jacobians of $K$-quasiconformal maps are Muckenhoupt weights belonging to the class $A_{p}$ for any $p>K$ (see [2, Theorem 13.4.2], or also [3]), and this is sharp. As a consequence of Theorem 1, we obtain the following improvement. 
Corollary 4. Let $\mu \in V M O$ be compactly supported, such that $\|\mu\|_{\infty}<1$, and let $\phi: \mathbf{C} \rightarrow \mathbf{C}$ be a quasiconformal solution of

$$
\bar{\partial} \phi(z)-\mu(z) \partial \phi(z)=0
$$

Then, for every $1<p<\infty$ there exists a constant $C=C(p) \geq 1$ such that the estimate

$$
\left(f_{D} J(z, \phi)^{p} \mathrm{~d} z\right)^{\frac{1}{p}} \leq C_{p} f_{D} J(z, \phi) \mathrm{d} z,
$$

holds for every disk $D \subset \mathbf{C}$.

By quasiconformality, the above result is equivalent to say that the inverse mapping $\phi^{-1}$ has jacobian determinant $J\left(\cdot, \phi^{-1}\right) \in A_{p}$ for every $p>1$. In turn, Johnson and Neugebauer [10] proved that this is equivalent to the fact that the composition with $\phi^{-1}$ quantitatively preserves the Muckenhoupt class $A_{2}$, and this is what we actually prove. The above Corollary improves the results in [9], which assert that $J(\cdot, \phi) \in L_{l o c}^{p}$ for every finite $p>1$. Note also that general $K$-quasiconformal maps need not satisfy the estimate (4) if $p \geq \frac{K}{K-1}$ [3].

The paper is structured as follows. In Section 2 we prove Theorem 2. In Section 3 we prove Theorem 1 and its counterpart for the generalized Beltrami equation. In Section 4 we study some applications. By $C$ we denote a positive constant that may change at each occurrence. $B(x, r)$ denotes the open ball with center $x$ and radius $r$, and $2 B$ means the open ball concentric with $B$ and having double radius.

\section{Compactness of commutators}

By singular integral operator $T$, we mean a linear operator on $L^{p}\left(\mathbf{R}^{n}\right)$ that can be written as

$$
T f(x)=\int_{\mathbf{R}^{n}} f(y) K(x, y) \mathrm{d} y .
$$

Here $K: \mathbf{R}^{n} \times \mathbf{R}^{n} \backslash\{x=y\} \rightarrow \mathbf{C}$ obeys the bounds

(1) $|K(x, y)| \leq \frac{C_{1}}{|x-y|^{n}}$,

(2) $\left|K(x, y)-K\left(x, y^{\prime}\right)\right| \leq C_{2} \frac{\left|y-y^{\prime}\right|}{|x-y|^{n+1}}$ whenever $|x-y| \geq 2\left|y-y^{\prime}\right|$,

(3) $\left|K(x, y)-K\left(x^{\prime}, y\right)\right| \leq C_{3} \frac{\left|x-x^{\prime}\right|}{|x-y|^{n+1}}$ whenever $|x-y| \geq 2\left|x-x^{\prime}\right|$.

One then calls $\|T\|_{C Z}=\max \left\{C_{1}, C_{2}, C_{3}\right\}$ the Calderón-Zygmund constant of $T$. Given a singular integral operator $T$, we define the truncated singular integral as

$$
T_{\epsilon} f(x)=\int_{|x-y| \geq \epsilon} K(x, y) f(y) \mathrm{d} y
$$

and the maximal singular integral by the relationship

$$
T_{*} f(x)=\sup _{\epsilon>0}\left|T_{\epsilon} f(x)\right| .
$$

As usually, we denote $f_{E} f(x) \mathrm{d} x=\frac{1}{|E|} \int_{E} f(x) \mathrm{d} x$. A weight is a function $\omega \in$ $L_{\text {loc }}^{1}\left(\mathbf{R}^{n}\right)$ such that $\omega(x)>0$ almost everywhere. A weight $\omega$ is said to belong to the Muckenhoupt class $A_{p}, 1<p<\infty$, if

$$
[\omega]_{A_{p}}:=\sup \left(f_{Q} \omega(x) \mathrm{d} x\right)\left(f_{Q} \omega(x)^{-\frac{p^{\prime}}{p}} \mathrm{~d} x\right)^{\frac{p}{p^{\prime}}}<\infty,
$$


where the supremum is taken over all cubes $Q \subset \mathbf{R}^{n}$, and where $\frac{1}{p}+\frac{1}{p^{\prime}}=1$. One may equivalently consider balls instead of cubes. By $L^{p}(\omega)$ we denote the set of measurable functions $f$ that satisfy

$$
\|f\|_{L^{p}(\omega)}=\left(\int_{\mathbf{R}^{n}}|f(x)|^{p} \omega(x) \mathrm{d} x\right)^{\frac{1}{p}}<\infty .
$$

The quantity $\|f\|_{L^{p}(\omega)}$ defines a complete norm in $L^{p}(\omega)$. It is well know that if $T$ is a Calderón-Zygmund operator, then $T$ and also $T_{*}$ are bounded in $L^{p}(\omega)$ whenever $\omega \in A_{p}$ (see for instance [7, Cap. IV, Theorems 3.1 and 3.6]). Also the HardyLittlewood maximal operator $M$ is bounded in $L^{p}(\omega)$. For more about $A_{p}$ classes and weighted spaces $L^{p}(\omega)$, we refer the reader to [7].

We first show the following sufficient condition for compactness in $L^{p}(\omega), \omega \in A_{p}$. Remember that a metric space $X$ is totally bounded if for every $\epsilon>0$ there exists a finite number of open balls of radius $\epsilon$ whose union is the space $X$. In addition, a metric space is compact if and only if it is complete and totally bounded.

Theorem 5. Let $p \in(1, \infty), \omega \in A_{p}$, and let $\mathfrak{F} \subset L^{p}(\omega)$. Then $\mathfrak{F}$ is totally bounded if it satisfies the next three conditions:

(1) $\mathfrak{F}$ is uniformly bounded, i.e. $\sup _{f \in \mathfrak{F}}\|f\|_{L^{p}(\omega)}<\infty$.

(2) $\mathfrak{F}$ is uniformly equicontinuous, i.e. $\sup _{f \in \mathfrak{F}}\|f(\cdot+h)-f(\cdot)\|_{L^{p}(\omega)} \stackrel{h \rightarrow 0}{\longrightarrow} 0$.

(3) $\mathfrak{F}$ uniformly vanishes at infinity, i.e. $\sup _{f \in \mathfrak{F}}\left\|f-\chi_{Q(0, R)} f\right\|_{L^{p}(\omega)} \stackrel{R \rightarrow \infty}{\longrightarrow} 0$, where $Q(0, R)$ is the cube with center at the origin and sidelength $2 R$.

Let us emphasize that Theorem 5 is a strong sufficient condition for compactness in $L^{p}(\omega)$, because for a general weight $\omega \in A_{p}$ the space $L^{p}(\omega)$ is not invariant under translations. Theorem 5 is proved by adapting the arguments in [8]. In particular, the following result (which can be found in [8, Lemma 1]) is essential.

Lemma 6. Let $X$ be a metric space. Suppose that for every $\epsilon>0$ one can find a number $\delta>0$, a metric space $W$ and an mapping $\Phi: X \rightarrow W$ such that $\Phi(X)$ is totally bounded, and the implication

$$
d(\Phi(x), \Phi(y))<\delta \quad \Longrightarrow \quad d(x, y)<\epsilon
$$

holds for any $x, y \in X$. Then $X$ is totally bounded.

Proof of Theorem 5. Suppose that the family $\mathfrak{F}$ satisfies the three conditions of Theorem 5, and let $\epsilon>0$ be fixed. According to the third assumption on $\mathfrak{F}$, we can choose a positive quantity $R>0$ such that

$$
\sup _{f \in \mathfrak{F}}\left\|f-f \chi_{Q(0, R)}\right\|_{L^{p}(\omega)}<\frac{\epsilon}{4}
$$

Let us also find $\rho>0$ small enough so that

$$
\sup _{h \in Q(0,2 \rho)}\left(\sup _{f \in \mathfrak{F}}\|f(\cdot)-f(\cdot+h)\|_{L^{p}(\omega)}\right)<\frac{\epsilon}{2^{2+n / p}} .
$$

Such a $\rho$ exists due to the equicontinuity assumption on $\mathfrak{F}$. Now, let us choose $N$ cubes $Q_{1}, \ldots, Q_{N}$ with sidelength $2 \rho$, having pairwise disjoint interiors, and such that

$$
\overline{Q(0, R)} \subset \overline{\bigcup_{i} Q_{i}}
$$


Define

$$
\Phi f(x)= \begin{cases}f_{Q_{i}} f(z) \mathrm{d} z, & x \in Q_{i}, i=1, \ldots, N \\ 0, & \text { otherwise. }\end{cases}
$$

Since functions in $L^{p}(\omega)$ are locally integrable, $\Phi f$ is well defined for any $f \in \mathfrak{F}$. Moreover,

$$
\begin{aligned}
\int_{\mathbf{R}^{n}}|\Phi f(x)|^{p} \omega(x) \mathrm{d} x & =\sum_{i=1}^{N}\left|f_{Q_{i}} f(z) \mathrm{d} z\right|^{p} \int_{Q_{i}} \omega(x) \mathrm{d}(x) \\
& \leq \sum_{i=1}^{N}\left(f_{Q_{i}}|f(z)|^{p} \omega(z) \mathrm{d} z\right)\left(f_{Q_{i}} \omega^{\frac{-p^{\prime}}{p}}(z) \mathrm{d} z\right)^{\frac{p}{p^{\prime}}} \int_{Q_{i}} \omega(x) \mathrm{d} x \\
& \leq[\omega]_{A_{p}}\|f\|_{L^{p}(\omega)}^{p} .
\end{aligned}
$$

In particular, $\Phi: L^{p}(\omega) \rightarrow L^{p}(\omega)$ is a bounded operator. As $\mathfrak{F}$ is bounded, then $\Phi(\mathfrak{F})$ is a bounded subset of a finite dimensional Banach space, and hence $\Phi(\mathfrak{F})$ is totally bounded.

On the other hand, by (7) and (9) one gets that

$$
\left\|f \chi_{\mathbf{R}^{n} \backslash \cup_{i} Q_{i}}\right\|_{L^{p}(\omega)} \leq\left\|f \chi_{\mathbf{R}^{n} \backslash Q(0, R)}\right\|_{L^{p}(\omega)}<\frac{\epsilon}{4},
$$

for any $f \in \mathfrak{F}$. Also, by Jensen's inequality,

$$
\begin{aligned}
\left\|f \chi_{\cup_{i} Q_{i}}-\Phi f\right\|_{L^{p}(\omega)}^{p} & =\sum_{i=1}^{N} \int_{Q_{i}}\left|f(x)-f_{Q_{i}} f(z) \mathrm{d} z\right|^{p} \omega(x) \mathrm{d} x \\
& \leq \sum_{i=1}^{N} \frac{1}{\left|Q_{i}\right|} \int_{Q_{i}} \int_{Q_{i}}|f(x)-f(z)|^{p} \mathrm{~d} z \omega(x) \mathrm{d} x .
\end{aligned}
$$

Now, if $x, z \in Q_{i}$, then $z-x=h \in Q(0,2 \rho)$. Therefore, after a change of coordinates,

$$
\begin{aligned}
\left\|f \chi_{\cup_{i} Q_{i}}-\Phi f\right\|_{L^{p}(\omega)}^{p} & \leq \sum_{i=1}^{N} \frac{1}{\left|Q_{i}\right|} \int_{Q_{i}} \int_{Q(0,2 \rho)}|f(x)-f(x+h)|^{p} \mathrm{~d} h \omega(x) \mathrm{d} x \\
& =\frac{1}{|Q(0, \rho)|} \int_{Q(0,2 \rho)} \sum_{i=1}^{N} \int_{Q_{i}}|f(x)-f(x+h)|^{p} \omega(x) \mathrm{d} x \mathrm{~d} h \\
& \leq \frac{1}{|Q(0, \rho)|} \int_{Q(0,2 \rho)} \int_{\mathbf{R}^{n}}|f(x)-f(x+h)|^{p} \omega(x) \mathrm{d} x \mathrm{~d} h \\
& =2^{n} f_{Q(0,2 \rho)}\|f(\cdot)-f(\cdot+h)\|_{L^{p}(\omega)}^{p} \mathrm{~d} h \\
& \leq 2^{n} \sup _{h \in Q(0,2 \rho)}\left(\sup _{f \in \mathfrak{F}}\|f(\cdot)-f(\cdot+h)\|_{L^{p}(\omega)}^{p}\right)<\left(\frac{\epsilon}{4}\right)^{p} .
\end{aligned}
$$

Summarizing,

$$
\|f-\Phi f\|_{L^{p}(\omega)} \leq\left\|f \chi_{\mathbf{R}^{n} \backslash \cup_{i} Q_{i}}\right\|_{L^{p}(\omega)}+\left\|f \chi_{\cup_{i} Q_{i}}-\Phi f\right\|_{L^{p}(\omega)}<\frac{\epsilon}{2},
$$


for any $f \in \mathfrak{F}$. Hence

$$
\|f\|_{L^{p}(\omega)}<\frac{\epsilon}{2}+\|\Phi f\|_{L^{p}(\omega)}, \quad \text { whenever } f \in \mathfrak{F} .
$$

Since $\Phi$ is linear, this means that

$$
\|f-g\|_{L^{p}(\omega)}<\frac{\epsilon}{2}+\|\Phi f-\Phi g\|_{L^{p}(\omega)}, \quad \text { whenever } f, g \in \mathfrak{F} .
$$

Set $\delta=\epsilon / 2$. The above inequality says that if $f, g \in \mathfrak{F}$ are such that $d(\Phi f, \Phi g)<\delta$, then $d(f, g)<\epsilon$. By the previous Lemma, it follows that $\mathfrak{F}$ is totally bounded.

In order to prove Theorem 2 , we will first reduce ourselves to smooth symbols $b$. Let us recall that commutators $C_{b}=[b, T]$ with $b \in B M O\left(\mathbf{R}^{n}\right)$ are continuous in $L^{p}(\omega)$ [15, Theorem 2.3]. Moreover, in [13, Theorem 1] the following estimate is shown,

$$
\left\|C_{b} f\right\|_{L^{p}(\omega)} \leq C\|b\|_{*}\left\|M^{2} f\right\|_{L^{p}(\omega)},
$$

where $\|b\|_{*}$ denotes the $B M O$ norm of $b$, and the constant $C$ may depend on $\omega$, but not on $b$. Now, by the boudedness of the Hardy-Littlewood operator $M$ on $L^{p}(\omega)$, we obtain

$$
\left\|C_{b} f\right\|_{L^{p}(\omega)} \leq C\|b\|_{*}\|f\|_{L^{p}(\omega)} .
$$

Since by assumption $b \in V M O\left(\mathbf{R}^{n}\right)$, we can approximate the function $b$ by functions $b_{j} \in \mathcal{C}_{c}^{\infty}\left(\mathbf{R}^{n}\right)$ in the $B M O$ norm, and thus

$$
\left\|C_{b} f-C_{b_{j}} f\right\|_{L^{p}(\omega)}=\left\|C_{b-b_{j}} f\right\|_{L^{p}(\omega)} \leq C\left\|b-b_{j}\right\|_{*}\|f\|_{L^{p}(\omega)} .
$$

In particular, the commutators with smooth symbol $C_{b_{j}}$ converge to $C_{b}$ in the operator norm of $L^{p}(\omega)$. Therefore it suffices to prove compactness for the commutator with smooth symbol.

Another reduction in the proof of Theorem 2 will be made by slightly modifying the singular integral operator $T$. This technique comes from Krantz and Li [12]. More precisely, for every $\eta>0$ small enough, let us take a continuous function $K^{\eta}$ defined on $\mathbf{R}^{n} \times \mathbf{R}^{n}$, taking values in $\mathbf{R}$ or $\mathbf{C}$, and such that:

(1) $K^{\eta}(x, y)=K(x, y)$ if $|x-y| \geq \eta$,

(2) $\left|K^{\eta}(x, y)\right| \leq \frac{C_{0}}{|x-y|^{n}}$ for $\frac{\eta}{2}<|x-y|<\eta$,

(3) $K^{\eta}(x, y)=0$ if $|x-y| \leq \frac{\eta}{2}$

where $C_{0}$ is independent of $\eta$. Due to the growth properties of $K$, it is not restrictive to suppose that condition 2 holds for all $x, y \in \mathbf{R}^{n}$. Now, let

$$
T^{\eta} f(x)=\int_{\mathbf{R}^{n}} K^{\eta}(x, y) f(y) \mathrm{d} y,
$$

and let us also denote

$$
C_{b}^{\eta} f(x)=\left[b, T^{\eta}\right] f(x)=\int_{\mathbf{R}^{n}}(b(x)-b(y)) K^{\eta}(x, y) f(y) \mathrm{d} y .
$$

We now prove that the commutators $C_{b}^{\eta}$ approximate $C_{b}$ in the operator norm.

Lemma 7. Let $b \in \mathcal{C}_{c}^{1}\left(\mathbf{R}^{n}\right)$. There exists a constant $C=C\left(n, C_{0}\right)$ such that

$$
\left|C_{b} f(x)-C_{b}^{\eta} f(x)\right| \leq C \eta\|\nabla b\|_{\infty} M f(x) \text { almost everywhere, }
$$

for every $\eta>0$. As a consequence,

$$
\lim _{\eta \rightarrow 0}\left\|C_{b}^{\eta}-C_{b}\right\|_{L^{p}(\omega) \rightarrow L^{p}(\omega)}=0
$$


whenever $\omega \in A_{p}$ and $1<p<\infty$.

Proof. Let $f \in L^{p}(\omega)$. For every $x \in \mathbf{R}^{n}$ we have

$$
\begin{aligned}
C_{b} f(x)-C_{b}^{\eta} f(x)= & \int_{|x-y|<\eta}(b(x)-b(y)) K(x, y) f(y) \mathrm{d} y \\
& -\int_{\frac{\eta}{2} \leq|x-y|<\eta}(b(x)-b(y)) K^{\eta}(x, y) f(y) \mathrm{d} y \\
= & I_{1}(x)+I_{2}(x) .
\end{aligned}
$$

Using the smoothness of $b$ and the size estimates of $K^{\eta}$, we have that

$$
\begin{aligned}
\left|I_{1}(x)\right| & \leq \int_{|x-y|<\eta}|b(y)-b(x)||K(x, y)||f(y)| \mathrm{d} y \\
& \leq C_{0}\|\nabla b\|_{\infty} \sum_{j=0}^{\infty} \int_{\frac{\eta}{2^{j+1}}<|x-y|<\frac{\eta}{2^{j}}} \frac{|f(y)|}{|x-y|^{n-1}} \mathrm{~d} y \\
& \leq 2^{n} C_{0}\|\nabla b\|_{\infty} \sum_{j=0}^{\infty} \frac{\eta|B(0,1)|}{2^{j+1}} f_{|x-y|<\frac{\eta}{2^{j}}}|f(y)| \mathrm{d} y \\
& \leq \eta 2^{n} C_{0}\|\nabla b\|_{\infty}|B(0,1)| M f(x)
\end{aligned}
$$

for almost every $x$. For the other term, similarly

$$
\begin{aligned}
\left|I_{2}(x)\right| & \leq \eta\|\nabla b\|_{\infty} \int_{\frac{\eta}{2}<|x-y|<\eta}\left|K^{\eta}(x, y)\right||f(y)| \mathrm{d} y \\
& \leq \eta C_{0}\|\nabla b\|_{\infty} \int_{\frac{\eta}{2}<|x-y|<\eta} \frac{|f(y)|}{|x-y|^{n}} \mathrm{~d} y \\
& \leq \eta 2^{n} C_{0}\|\nabla b\|_{\infty}|B(0,1)| f_{|x-y|<\eta}|f(y)| \mathrm{d} y \\
& \leq \eta 2^{n} C_{0}\|\nabla b\|_{\infty}|B(0,1)| M f(x) .
\end{aligned}
$$

Therefore, the pointwise estimate follows. Now, the boundedness of $M$ in $L^{p}(\omega)$ for any $A_{p}$ weight $\omega$ implies that

$$
\left\|C_{b} f-C_{b}^{\eta} f\right\|_{L^{p}(\omega)} \leq C \eta\|\nabla b\|_{\infty}\|M f\|_{L^{p}(\omega)} \leq C \eta\|\nabla b\|_{\infty}\|f\|_{L^{p}(\omega)} \rightarrow 0,
$$

as $\eta \rightarrow 0$. This finishes the proof of Lemma 7 .

We are now ready to conclude the proof of Theorem 2. From now on, $\eta>0$ and $b \in \mathcal{C}_{c}^{1}\left(\mathbf{R}^{n}\right)$ are fixed, and we have to prove that the commutator $C_{b}^{\eta}=\left[b, T^{\eta}\right]$ is compact. Thus, the constants that will appear may depend on $b$ and $\eta$.

We denote $\mathfrak{F}=\left\{C_{b}^{\eta} f ; f \in L^{p}(\omega),\|f\|_{L^{p}(\omega)} \leq 1\right\}$. Then $\mathfrak{F}$ is uniformly bounded, because $C_{b}^{\eta}$ is a bounded operator on $L^{p}(\omega)$. To prove the uniform equicontinuity of $\mathfrak{F}$, we must see that

$$
\lim _{h \rightarrow 0} \sup _{f \in \mathfrak{F}}\left\|C_{b}^{\eta} f(\cdot)-C_{b}^{\eta} f(\cdot+h)\right\|_{L^{p}(\omega)}=0 .
$$


To do this, let us write

$$
\begin{aligned}
C_{b}^{\eta} f(x)-C_{b}^{\eta} f(x+h)= & (b(x)-b(x+h)) \int_{\mathbf{R}^{n}} K^{\eta}(x, y) f(y) \mathrm{d} y \\
& +\int_{\mathbf{R}^{n}}(b(x+h)-b(y))\left(K^{\eta}(x, y)-K^{\eta}(x+h, y)\right) f(y) \mathrm{d} y \\
= & \int_{\mathbf{R}^{n}} I_{1}(x, y, h) \mathrm{d} y+\int_{\mathbf{R}^{n}} I_{2}(x, y, h) \mathrm{d} y .
\end{aligned}
$$

For $I_{1}(x, y, h)$, using the regularity of the function $b$ and the definition of the operator $T_{*}$,

$$
\begin{aligned}
& \left|\int_{\mathbf{R}^{n}} I_{1}(x, y, h) \mathrm{d} y\right| \\
& \leq\|\nabla b\|_{\infty}|h|\left|\int_{|x-y|>\frac{\eta}{2}}\left(K^{\eta}(x, y)-K(x, y)\right) f(y) \mathrm{d} y+\int_{|x-y|>\frac{\eta}{2}} K(x, y) f(y) \mathrm{d} y\right| \\
& \leq\|\nabla b\|_{\infty}|h|\left(\int_{|x-y|>\frac{\eta}{2}}\left|K^{\eta}(x, y)-K(x, y)\right||f(y)| \mathrm{d} y+T_{*} f(x)\right) \\
& \leq\|\nabla b\|_{\infty}|h|\left(C M f(x)+T_{*} f(x)\right)
\end{aligned}
$$

for some constant $C>0$ that may depend on $\eta$, but not on $h$. Therefore

$$
\left(\int\left|\int_{\mathbf{R}^{n}} I_{1}(x, y, h) \mathrm{d} y\right|^{p} \omega(x) \mathrm{d} x\right)^{\frac{1}{p}} \leq C|h|\|f\|_{L^{p}(\omega)},
$$

for $C$ independent of $f$ and $h$. Here we used the boundedness of $M$ and $T_{*}$ on $L^{p}(\omega)$ (see [7, Chap. IV, Th. 3.6]). We will divide the integral of $I_{2}(x, y, h)$ into three regions:

$$
\begin{aligned}
& A=\left\{y \in \mathbf{R}^{n}:|x-y|>\frac{\eta}{2},|x+h-y|>\frac{\eta}{2}\right\}, \\
& B=\left\{y \in \mathbf{R}^{n}:|x-y|>\frac{\eta}{2},|x+h-y|<\frac{\eta}{2}\right\}, \\
& C=\left\{y \in \mathbf{R}^{n}:|x-y|<\frac{\eta}{2},|x+h-y|>\frac{\eta}{2}\right\} .
\end{aligned}
$$

Note that $I_{2}(x, y, h)=0$ for $y \in \mathbf{R}^{n} \backslash A \cup B \cup C$. Now, for the integral over $A$, we use the smoothness of $b$ and $K^{\eta}$,

$$
\begin{aligned}
\left|\int_{A} I_{2}(x, y, h) \mathrm{d} y\right| & \leq C\|\nabla b\|_{\infty}|h| \int_{|x-y|>\frac{\eta}{4}} \frac{|f(y)|}{|x-y|^{n+1}} \mathrm{~d} y \\
& \leq C\|\nabla b\|_{\infty} \frac{|h|}{\eta} \sum_{j=0}^{\infty} 2^{-j} f_{|x-y|<\frac{2^{j} \eta}{4}}|f(y)| \mathrm{d} y \leq C\|\nabla b\|_{\infty} \frac{|h|}{\eta} \quad \operatorname{Mf}(x),
\end{aligned}
$$

thus

$$
\left(\int_{\mathbf{R}^{n}}\left|\int_{A} I_{2}(x, y, h) \mathrm{d} y\right|^{p} \omega(x) \mathrm{d} x\right)^{\frac{1}{p}} \leq C|h|\|f\|_{L^{p}(\omega)} .
$$

for some constant $C$ that may depend on $\eta$ and $b$, but not on $h$. In particular, the term on the right hand side goes to 0 as $|h| \rightarrow 0$. 
The integrals of $I_{2}(x, y, h)$ over $B$ and $C$ are symmetric, so we only give the details once. For the integral over the set $B$, let us assume that $|h|$ is very small. We can first choose $R_{0}>\eta / 2+|h|$ such that $b$ vanishes outside the ball $B_{0}=B\left(0, R_{0}\right)$. It then follows that $b(\cdot+h)$ has support in $2 B_{0}$. Then, since $B \subset B(x,|h|+\eta / 2)$, we have for $|x|<3 R_{0}$ that $B \subset 4 B_{0}$ and therefore

$$
\begin{aligned}
\left|\int_{B} I_{2}(x, y, h) \mathrm{d} y\right| & \leq C_{0}\|\nabla b\|_{\infty} \int_{B \cap 4 B_{0}} \frac{|x+h-y||f(y)|}{|x-y|^{n}} \mathrm{~d} y \\
& \leq C_{0}\|\nabla b\|_{\infty} \int_{B \cap 4 B_{0}} \frac{|f(y)|}{|x-y|^{n-1}} \mathrm{~d} y \\
& \leq C_{0}\|\nabla b\|_{\infty}(2 / \eta)^{n-1} \int_{B \cap 4 B_{0}}|f(y)| \omega(y)^{\frac{1}{p}} \omega(y)^{-\frac{1}{p}} \mathrm{~d} y \\
& \leq C_{0}\|\nabla b\|_{\infty}(2 / \eta)^{n-1}\|f\|_{L^{p}(\omega)}\left(\int_{B \cap 4 B_{0}} \omega(y)^{-\frac{p^{\prime}}{p}} \mathrm{~d} y\right)^{\frac{1}{p^{\prime}}}
\end{aligned}
$$

whence

$$
\int_{3 B_{0}}\left|\int_{B} I_{2}(x, y, h) \mathrm{d} y\right|^{p} \omega(x) \mathrm{d} x \leq C\|f\|_{L^{p}(\omega)}^{p}\left(\int_{3 B_{0}} \omega(x) \mathrm{d} x\right)\left(\int_{B \cap 4 B_{0}} \omega(y)^{-\frac{p^{\prime}}{p}} \mathrm{~d} y\right)^{\frac{p}{p^{\prime}}}
$$

for some constant $C$ that might depend on $\eta$, but not on $h$. If, instead, we have $|x| \geq 3 R_{0}$, then $b(x+h)=0$ (because $|h|<R_{0}$ so that $\left.|x+h|>2 R_{0}\right)$. Note also that for $y \in B$ one has $|x| \leq C|x-y|$ where $C$ depends only on $\eta$. Therefore

$$
\begin{aligned}
\left|\int_{B} I_{2}(x, y, h) \mathrm{d} y\right| & \leq C\|b\|_{\infty} \int_{B \cap 4 B_{0}} \frac{|f(y)|}{|x-y|^{n}} \mathrm{~d} y \leq \frac{C\|b\|_{\infty}}{|x|^{n}} \int_{B \cap 4 B_{0}}|f(y)| \mathrm{d} y \\
& \leq \frac{C\|b\|_{\infty}}{|x|^{n}}\|f\|_{L^{p}(\omega)}\left(\int_{B \cap 4 B_{0}} \omega(y)^{-\frac{p^{\prime}}{p}} \mathrm{~d} y\right)^{\frac{1}{p^{\prime}}} .
\end{aligned}
$$

This implies that

$$
\begin{aligned}
& \int_{\mathbf{R}^{n} \backslash 3 B_{0}}\left|\int_{B} I_{2}(x, y, h) \mathrm{d} y\right|^{p} \omega(x) \mathrm{d} x \\
& \leq C\|b\|_{\infty}^{p}\|f\|_{L^{p}(\omega)}^{p}\left(\int_{\mathbf{R}^{n} \backslash 3 B_{0}} \frac{\omega(x)}{|x|^{n p}} \mathrm{~d} x\right)\left(\int_{B \cap 4 B_{0}} \omega(y)^{-\frac{p^{\prime}}{p}} \mathrm{~d} y\right)^{\frac{p}{p^{\prime}}} .
\end{aligned}
$$

Summarizing,

$$
\begin{aligned}
& \int_{\mathbf{R}^{n}}\left|\int_{B} I_{2}(x, y, h) \mathrm{d} y\right|^{p} \omega(x) \mathrm{d} x \\
& \leq C\|f\|_{L^{p}(\omega)}^{p}\left(\int_{B \cap 4 B_{0}} \omega(y)^{-\frac{p^{\prime}}{p}} \mathrm{~d} y\right)^{\frac{p}{p^{\prime}}}\left(\int_{3 B_{0}} \omega(x) \mathrm{d} x+\int_{\mathbf{R}^{n} \backslash 3 B_{0}} \frac{\omega(x)}{|x|^{n p}} \mathrm{~d} x\right) .
\end{aligned}
$$

After proving that

$$
\int_{|x|>3 R_{0}} \frac{\omega(x)}{|x|^{n p}} \mathrm{~d} x<\infty
$$

the left hand side of (14) will converge to 0 as $|h| \rightarrow 0$ since $|B| \rightarrow 0$ as $|h| \rightarrow 0$. To prove the above claim, let us choose $q<p$ such that $\omega \in A_{q}[7$, Theorem 2.6, 
Ch. IV]. For such $q$, we have

$$
\int_{|x|>R} \frac{\omega(x)}{|x|^{n p}} \mathrm{~d} x=\sum_{j=1}^{\infty} \int_{2^{j-1}<\frac{|x|}{R}<2^{j}} \frac{\omega(x)}{|x|^{n p}} \mathrm{~d} x \leq \sum_{j=1}^{\infty}\left(2^{j-1} R\right)^{-n p} \omega\left(B\left(0,2^{j} R\right)\right) .
$$

By [7, Lemma 2.2], we have

$$
\int_{|x|>R} \frac{\omega(x)}{|x|^{n p}} \mathrm{~d} x \leq \sum_{j=1}^{\infty}\left(2^{j-1} R\right)^{-n p}\left(2^{j} R\right)^{n q} \omega(B(0,1))=\frac{C}{R^{n(p-q)}}<\infty
$$

as desired. The equicontinuity of $\mathfrak{F}$ follows.

Finally, we show the decay at infinity of the elements of $\mathfrak{F}$. Let $x$ be such that $|x|>R>R_{0}$. Then, $x \notin \operatorname{supp} b$, and

$$
\begin{aligned}
\left|C_{b}^{\eta} f(x)\right| & =\left|\int_{\mathbf{R}^{n}}(b(x)-b(y)) K^{\eta}(x, y) f(y) \mathrm{d} y\right| \leq C_{0}\|b\|_{\infty} \int_{\operatorname{supp} b} \frac{|f(y)|}{|x-y|^{n}} \mathrm{~d} y \\
& \leq \frac{C\|b\|_{\infty}}{|x|^{n}} \int_{\operatorname{supp} b}|f(y)| \mathrm{d} y \leq \frac{C\|b\|_{\infty}}{|x|^{n}}\|f\|_{L^{p}(\omega)}\left(\int_{\operatorname{supp} b} \omega(y)^{-\frac{p^{\prime}}{p}} d y\right)^{\frac{1}{p^{\prime}}}
\end{aligned}
$$

whence

$$
\left(\int_{|x|>R}\left|C_{b}^{\eta} f(x)\right|^{p} \omega(x) \mathrm{d} x\right)^{\frac{1}{p}} \leq C\|b\|_{\infty}\|f\|_{L^{p}(\omega)}\left(\int_{|x|>R} \frac{\omega(x)}{|x|^{n p}} \mathrm{~d} x\right)^{\frac{1}{p}} .
$$

The right hand side above converges to 0 as $R \rightarrow \infty$, due to (15). By Theorem $5, \mathfrak{F}$ is totally bounded. Theorem 2 follows.

\section{A priori estimates for Beltrami equations}

We first prove Theorem 1. To do this, let us remember that the Beurling-Ahlfors singular integral operator is defined by the following principal value

$$
\mathcal{B} f(z)=-\frac{1}{\pi} P . V \cdot \int \frac{f(w)}{(z-w)^{2}} \mathrm{~d} w .
$$

This operator can be seen as the formal $\partial$ derivative of the Cauchy transform,

$$
\mathcal{C} f(z)=\frac{1}{\pi} \int \frac{f(w)}{z-w} \mathrm{~d} w
$$

At the frequency side, $\mathcal{B}$ corresponds to the Fourier multiplier $m(\xi)=\frac{\bar{\xi}}{\xi}$, so that $\mathcal{B}$ is an isometry in $L^{2}(\mathbf{C})$. Moreover, this Fourier representation also explains the important relation

$$
\mathcal{B}(\bar{\partial} f)=\partial f
$$

for smooth enough functions $f$. By $\mathcal{B}^{*}$ we mean the singular integral operator obtained by simply conjugating the kernel of $\mathcal{B}$, that is,

$$
\mathcal{B}^{*}(f)(z)=-\frac{1}{\pi} P . V \cdot \int \frac{f(w)}{(\bar{z}-\bar{w})^{2}} \mathrm{~d} w .
$$

Note that $\mathcal{B}^{*}$ has Fourier multiplier $m^{*}(\xi)=\frac{\xi}{\xi}$. Thus,

$$
\mathcal{B B}^{*}=\mathcal{B}^{*} \mathcal{B}=\mathbf{I d}
$$


In other words, $\mathcal{B}^{*}$ is the $L^{2}$-inverse of $\mathcal{B}$. It also appears as the $\mathbf{C}$-linear adjoint of $\mathcal{B}$,

$$
\int_{\mathbf{C}} \mathcal{B} f(z) \overline{g(z)} \mathrm{d} z=\int_{\mathbf{C}} f(z) \overline{\mathcal{B}^{*} g(z)} \mathrm{d} z .
$$

The complex conjugate operator $\overline{\mathcal{B}}$ is the composition of $\mathcal{B}$ with the complex conjugation operator $\mathbf{C} f=\bar{f}$, that is,

$$
\overline{\mathcal{B}}(f)=\mathbf{C B}(f)=\overline{\mathcal{B}(f)} .
$$

It then follows that

$$
\overline{\mathcal{B}}=\mathbf{C B}=\mathcal{B}^{*} \mathbf{C}
$$

Note that $\mathcal{B}$ and $\mathcal{B}^{*}$ are $\mathbf{C}$-linear operators, while $\overline{\mathcal{B}}$ is only $\mathbf{R}$-linear. See [2, Chapter 4$]$ for more about the Beurling-Ahlfors transform.

Proof of Theorem 1. We follow Iwaniec's idea [9, pp. 42-43]. For every $N=$ $1,2, \ldots$, let

$$
P_{N}=\mathbf{I d}+\mu \mathcal{B}+\cdots+(\mu \mathcal{B})^{N}
$$

Then

$$
(\mathbf{I d}-\mu \mathcal{B}) P_{N-1}=P_{N-1}(\mathbf{I} \mathbf{d}-\mu \mathcal{B})=\mathbf{I} \mathbf{d}-\mu^{N} \mathcal{B}^{N}+K_{N},
$$

where $K_{N}=\mu^{N} \mathcal{B}^{N}-(\mu \mathcal{B})^{N}$. Each $K_{N}$ consists of a finite sum of operators that contain the commutator $[\mu, \mathcal{B}]$ as a factor. Thus, by Theorem 2 , each $K_{N}$ is compact in $L^{p}(\omega)$. On the other hand, the $N$-th iterate $\mathcal{B}^{N}$ of the Beurling transform is another convolution-type Calderón-Zygmund operator, whose kernel is

$$
b_{N}(z)=\frac{(-1)^{N} N}{\pi} \frac{\bar{z}^{N-1}}{z^{N+1}}
$$

(see for instance [16, p. 73]). Arguing as in [6, Lemma $7.9 \&$ Theorem 7.11], one sees that the operator norm $\left\|\mathcal{B}^{N}\right\|_{L^{p}(\omega)}$ depends linearly on both the unweighted norm $\left\|B^{N}\right\|_{L^{p}\left(\mathbf{R}^{n}\right)}$ and the Calderón-Zygmund constant $\left\|B^{N}\right\|_{C Z}$. Since both quantities are bounded by a constant multiple of $N^{2}$, one immediately sees that

$$
\left\|\mathcal{B}^{N}\right\|_{L^{p}(\omega)} \leq C N^{2}
$$

with constant $C$ that depends on $[\omega]_{A_{p}}$, but not on $N$. As a consequence,

$$
\left\|\mu^{N} \mathcal{B}^{N} f\right\|_{L^{p}(\omega)} \leq C N^{2}\|\mu\|_{\infty}^{N}\|f\|_{L^{p}(\omega)}
$$

and therefore, for large enough $N$, the operator Id $-\mu^{N} \mathcal{B}^{N}$ is invertible. This, together with (16), says that $\mathbf{I d}-\mu \mathcal{B}$ is an Fredholm operator. Now apply the index theory to $\mathbf{I d}-\mu \mathcal{B}$. The continuous deformation $\mathbf{I d}-t \mu \mathcal{B}, 0 \leq t \leq 1$, is a homotopy from the identity operator to $\mathbf{I d}-\mu \mathcal{B}$. By the homotopical invariance of Index,

$$
\operatorname{Index}(\mathbf{I d}-\mu \mathcal{B})=\operatorname{Index}(\mathbf{I d})=0 .
$$

Since injective operators with 0 index are onto, for the invertibility of $\mathbf{I d}-\mu \mathcal{B}$ it just remains to show that it is injective. So let $f \in L^{p}(\omega)$ be such that $f=\mu \mathcal{B} f$. Then $f$ has compact support. Now, since belonging to $A_{p}$ is an open-ended condition (see 
e.g. [7, Theorem IV.2.6]), there exists $\delta>0$ such that $p-\delta>1$ and $\omega \in A_{p-\delta}$. Then $\omega^{-\frac{1}{p-\delta}} \in L_{l o c}^{1}(\mathbf{C})$. Taking $\epsilon=\frac{\delta}{p-\delta}$, we obtain

$$
\begin{aligned}
\int_{\mathbf{C}}|f(x)|^{1+\epsilon} \mathrm{d} x & \leq\left(\int_{\operatorname{supp} f}|f(x)|^{p} \omega(x) \mathrm{d} x\right)^{\frac{1+\epsilon}{p}}\left(\int_{\operatorname{supp} f} \omega(x)^{-\frac{1+\epsilon}{p-(1+\epsilon)}} \mathrm{d} x\right)^{\frac{p-(1+\epsilon)}{p}} \\
& \leq\|f\|_{L^{p}(\omega)}^{1+\epsilon}\left(\int_{\operatorname{supp} f} \omega(x)^{-\frac{1+\epsilon}{p-(1+\epsilon)}} \mathrm{d} x\right)^{\frac{p-(1+\epsilon)}{p}}<\infty
\end{aligned}
$$

therefore $f \in L^{1+\epsilon}(\mathbf{C})$. But $\mathbf{I d}-\mu \mathcal{B}$ is injective on $L^{p}(\mathbf{C}), 1<p<\infty$, when $\mu \in V M O(\mathbf{C})$, by Iwaniec's Theorem. Hence, $f \equiv 0$.

Finally, since $\mathbf{I d}-\mu \mathcal{B}: L^{p}(\omega) \rightarrow L^{p}(\omega)$ is linear, bounded, and invertible, it then follows that it has a bounded inverse, so the inequality

$$
\|g\|_{L^{p}(\omega)} \leq C\|(\mathbf{I d}-\mu \mathcal{B}) g\|_{L^{p}(\omega)}
$$

holds for every $g \in L^{p}(\omega)$. Here the constant $C>0$ depends only on the $L^{p}(\omega)$ norm of $\mathbf{I d}-\mu \mathcal{B}$, and therefore on $p, k$ and $[\omega]_{A_{p}}$, but not on $g$. As a consequence, given $g \in L^{p}(\omega)$, and setting

$$
f:=\mathcal{C}(\mathbf{I d}-\mu \mathcal{B})^{-1} g,
$$

we immediately see that $f$ satisfies $\bar{\partial} f-\mu \partial f=g$. Moreover, since $\omega \in A_{p}$,

$$
\begin{aligned}
\|D f\|_{L^{p}(\omega)} & \leq\|\partial f\|_{L^{p}(\omega)}+\|\bar{\partial} f\|_{L^{p}(\omega)} \\
& =\left\|\mathcal{B}(\mathbf{I} \mathbf{d}-\mu \mathcal{B})^{-1} g\right\|_{L^{p}(\omega)}+\left\|(\mathbf{I} \mathbf{d}-\mu \mathcal{B})^{-1} g\right\|_{L^{p}(\omega)} \leq C\|g\|_{L^{p}(\omega)},
\end{aligned}
$$

where still $C$ depends only on $p, k$ and $[\omega]_{A_{p}}$.

For the uniqueness, let us choose two solutions $f_{1}, f_{2}$ to the inhomogeneous equation. The difference $F=f_{1}-f_{2}$ defines a solution to the homogeneous equation $\bar{\partial} F-\mu \partial F=0$. Moreover, one has that $D F \in L^{p}(\omega)$ and, arguing as before, one sees that $D F \in L^{1+\epsilon}(\mathbf{C})$. In particular, this says that $(I-\mu \mathcal{B})(\bar{\partial} F)=0$. But for $\mu \in V M O(\mathbf{C})$, it follows from Iwaniec's Theorem that $\mathbf{I d}-\mu \mathcal{B}$ is injective in $L^{p}(\mathbf{C})$ for any $1<p<\infty$, whence $\bar{\partial} F=0$. Thus $D F=0$ and so $F$ is a constant.

The $\mathbf{C}$-linear Beltrami equation is a particular case of the following one,

$$
\bar{\partial} f(z)-\mu(z) \partial f(z)-\nu(z) \overline{\partial f(z)}=g(z),
$$

which we will refer to as the generalized Beltrami equation. It is well known that, in the plane, any linear, elliptic system, with two unknowns and two first-order equations on the derivatives, reduces to the above equation (modulo complex conjugation), whence the interest in understanding it is very big. An especially interesting example is obtained by setting $\mu=0$, when one obtains the so-called conjugate Beltrami equation,

$$
\bar{\partial} f(z)-\nu(z) \overline{\partial f(z)}=g(z)
$$

A direct adaptation of the above proof immediately drives the problem towards the commutator $[\nu, \overline{\mathcal{B}}]$. Unfortunately, as an operator from $L^{p}(\omega)$ onto itself, such commutator is not compact in general, even when $\omega=1$. To show this, let us choose

$$
\nu=i \nu_{0} \chi_{\mathbf{D}}+\nu_{1} \chi_{\mathbf{C} \backslash \mathbf{D}}
$$


where the constant $\nu_{0} \in \mathbf{R}$ and the function $\nu_{1}$ are chosen so that $\nu$ is continuous on $\mathbf{C}$, compactly supported in $2 \mathbf{D}$, with $\|\nu\|_{\infty}<1$. Let us also consider

$$
E=\left\{f \in L^{p} ;\|f\|_{L^{p}} \leq 1, \operatorname{supp}(f) \subset \mathbf{D}\right\},
$$

which is a bounded subset of $L^{p}$. For every $f \in E$, one has

$$
\begin{aligned}
\nu \overline{\mathcal{B}(f)}-\overline{\mathcal{B}(\nu f)} & =\chi_{\mathbf{D}} i \nu_{0} \overline{\mathcal{B}(f)}+\chi_{\mathbf{C} \backslash \mathbf{D}} \nu_{1} \overline{\mathcal{B}(f)}-\overline{\mathcal{B}\left(i \nu_{0} f\right)} \\
& =\chi_{\mathbf{D}} i \nu_{0} \overline{\mathcal{B}(f)}+\chi_{\mathbf{C} \backslash \mathbf{D}} \nu_{1} \overline{\mathcal{B}(f)}+i \nu_{0} \overline{\mathcal{B}(f)} \\
& =\chi_{\mathbf{D}} 2 i \nu_{0} \overline{\mathcal{B}(f)}+\chi_{\mathbf{C} \backslash \mathbf{D}}\left(i \nu_{0}+\nu_{1}\right) \overline{\mathcal{B}(f)} .
\end{aligned}
$$

In view of this relation, and since $\mathcal{B}$ is not compact, we have just cooked a concrete example of function $\nu \in V M O$ for wich the commutator $[\nu, \overline{\mathcal{B}}]$ is not compact. Nevertheless, it turns out that still a priori estimates hold, even for the generalized equation.

Theorem 8. Let $1<p<\infty, \omega \in A_{p}$, and let $\mu, \nu \in V M O(\mathbf{C})$ be compactly supported, such that $\||\mu|+|\nu|\|_{\infty}<1$. Let $g \in L^{p}(\omega)$. Then the equation

$$
\bar{\partial} f(z)-\mu(z) \partial f(z)-\nu(z) \overline{\partial f(z)}=g(z)
$$

has a solution $f$ with $D f \in L^{p}(\omega)$ and

$$
\|D f\|_{L^{p}(\omega)} \leq C\|g\|_{L^{p}(\omega)} .
$$

This solution is unique, modulo an additive constant.

A previous proof for the above result has been shown in [11] for the constant weight $\omega=1$. For the weighted counterpart, the arguments are based on a Neumann series argument similar to that in [11], with some minor modification. We write it here for completeness. The following Lemma will be needed.

Lemma 9. Let $\mu, \nu \in L^{\infty}(\mathbf{C})$ be measurable, bounded with compact support, such that $\||\mu|+|\nu|\|_{\infty}<1$. If $1<p<\infty$ and $p^{\prime}=\frac{p}{p-1}$, then the following statements are equivalent:

(1) The operator $\mathbf{I d}-\mu \mathcal{B}-\nu \overline{\mathcal{B}}: L^{p}(\mathbf{C}) \rightarrow L^{p}(\mathbf{C})$ is bijective.

(2) The operator $\mathbf{I d}-\bar{\mu} \mathcal{B}^{*}-\nu \overline{\mathcal{B}^{*}}: L^{p^{\prime}}(\mathbf{C}) \rightarrow L^{p^{\prime}}(\mathbf{C})$ is bijective.

Proof. When $\nu=0$, the above result is well known, and follows as an easy consequence of the fact that, with respect to the dual pairing

$$
\langle f, g\rangle=\int_{\mathbf{C}} f(z) \overline{g(z)} \mathrm{d} z,
$$

the operator Id $-\mu \mathcal{B}: L^{p}(\mathbf{C}) \rightarrow L^{p}(\mathbf{C})$ has precisely $\mathbf{I d}-\mathcal{B}^{*} \bar{\mu}: L^{p^{\prime}}(\mathbf{C}) \rightarrow L^{p^{\prime}}(\mathbf{C})$ as its $\mathbf{C}$-linear adjoint. Unfortunately, when $\nu$ does not identically vanish, $\mathbf{R}$-linear operators do not have an adjoint with respect to this dual pairing. An alternative proof can be found in [11]. We will think the space of $\mathbf{C}$-valued $L^{p}$ functions $L^{p}(\mathbf{C})$ as an R-linear space,

$$
L^{p}(\mathbf{C})=L_{\mathbf{R}}^{p}(\mathbf{C}) \oplus L_{\mathbf{R}}^{p}(\mathbf{C}),
$$

by means of the obvious identification $u+i v=(u, v)$. According to this product structure, every bounded $\mathbf{R}$-linear operator $T: L_{\mathbf{R}}^{p}(\mathbf{C}) \oplus L_{\mathbf{R}}^{p}(\mathbf{C}) \rightarrow L_{\mathbf{R}}^{p}(\mathbf{C}) \oplus L_{\mathbf{R}}^{p}(\mathbf{C})$ has an obvious matrix representation

$$
T(u+i v)=T\left(\begin{array}{l}
u \\
v
\end{array}\right)=\left(\begin{array}{ll}
T_{11} & T_{12} \\
T_{21} & T_{22}
\end{array}\right)\left(\begin{array}{l}
u \\
v
\end{array}\right)
$$


where every $T_{i j}: L_{\mathbf{R}}^{p}(\mathbf{C}) \rightarrow L_{\mathbf{R}}^{p}(\mathbf{C})$ is bounded. Similarly, bounded linear functionals $U: L_{\mathbf{R}}^{p}(\mathbf{C}) \oplus L_{\mathbf{R}}^{p}(\mathbf{C}) \rightarrow \mathbf{R}$ are represented by

$$
U\left(\begin{array}{l}
u \\
v
\end{array}\right)=\left(\begin{array}{ll}
U_{1} & U_{2}
\end{array}\right)\left(\begin{array}{l}
u \\
v
\end{array}\right)
$$

where every $U_{j}: L_{\mathbf{R}}^{p}(\mathbf{C}) \rightarrow \mathbf{R}$ is bounded. By the Riesz Representation Theorem, we get that $L_{\mathbf{R}}^{p}(\mathbf{C}) \oplus L_{\mathbf{R}}^{p}(\mathbf{C})$ has precisely $L_{\mathbf{R}}^{p^{\prime}}(\mathbf{C}) \oplus L_{\mathbf{R}}^{p^{\prime}}(\mathbf{C})$ as its topological dual space. In fact, we have an $\mathbf{R}$-bilinear dual pairing,

$$
\left\langle\left(\begin{array}{l}
u \\
v
\end{array}\right),\left(\begin{array}{l}
u^{\prime} \\
v^{\prime}
\end{array}\right)\right\rangle=\int u(z) u^{\prime}(z) \mathrm{d} z+\int v(z) v^{\prime}(z) \mathrm{d} z
$$

whenever $(u, v) \in L_{\mathbf{R}}^{p}(\mathbf{C}) \oplus L_{\mathbf{R}}^{p}(\mathbf{C})$ and $\left(u^{\prime}, v^{\prime}\right) \in L_{\mathbf{R}}^{p^{\prime}}(\mathbf{C}) \oplus L_{\mathbf{R}}^{p^{\prime}}(\mathbf{C})$, and which is nothing but the real part of (19). Under this new dual pairing, every $\mathbf{R}$-linear opeartor $T: L_{\mathbf{R}}^{p}(\mathbf{C}) \oplus L_{\mathbf{R}}^{p}(\mathbf{C}) \rightarrow L_{\mathbf{R}}^{p}(\mathbf{C}) \oplus L_{\mathbf{R}}^{p}(\mathbf{C})$ can be associated another operator

$$
T^{\prime}: L_{\mathbf{R}}^{p^{\prime}}(\mathbf{C}) \oplus L_{\mathbf{R}}^{p^{\prime}}(\mathbf{C}) \rightarrow L_{\mathbf{R}}^{p^{\prime}}(\mathbf{C}) \oplus L_{\mathbf{R}}^{p^{\prime}}(\mathbf{C}),
$$

called the $\mathbf{R}$-adjoint operator of $T$, defined by the common rule

$$
\left\langle\left(\begin{array}{l}
u \\
v
\end{array}\right), T^{\prime}\left(\begin{array}{l}
u^{\prime} \\
v^{\prime}
\end{array}\right)\right\rangle=\left\langle T\left(\begin{array}{l}
u \\
v
\end{array}\right),\left(\begin{array}{c}
u^{\prime} \\
v^{\prime}
\end{array}\right)\right\rangle \text {. }
$$

If $T$ is a $\mathbf{C}$-linear operator, then $T^{\prime}$ is the same as the $\mathbf{C}$-adjoint $T^{*}$ (i.e. the adjoint with respect to (19)) so in particular for the Beurling-Ahlfors transform $\mathcal{B}$ we have an $\mathbf{R}$-adjoint $\mathcal{B}^{\prime}$, and moreover $\mathcal{B}^{*}=\mathcal{B}^{\prime}$. Similarly, the pointwise multiplication by $\mu$ and $\nu$ are also $\mathbf{C}$-linear operators. Thus their $\mathbf{R}$-adjoints $\mu^{\prime}, \nu^{\prime}$ agree with their respectives $\mathbf{C}$-adjoints $\mu^{*}, \nu^{*}$. But these are precisely the pointwise multiplication with the respective complex conjugates. Symbollically, $\mu^{\prime}=\bar{\mu}$ and $\nu^{\prime}=\bar{\nu}$. In contrast, general $\mathbf{R}$-linear operators need not have a $\mathbf{C}$-adjoint. For example, for the complex conjugation,

$$
\mathbf{C}=\left(\begin{array}{cc}
\mathbf{I d} & 0 \\
0 & -\mathbf{I d}
\end{array}\right)
$$

one simply has $\mathbf{C}^{\prime}=\mathbf{C}$. Putting all these things together, one easily sees that

$$
\begin{aligned}
(\mathbf{I} \mathbf{d}-\mu \mathcal{B}-\nu \overline{\mathcal{B}})^{\prime} & =(\mathbf{I} \mathbf{d}-\mu \mathcal{B}-\nu \mathbf{C B})^{\prime}=\mathbf{I} \mathbf{d}-(\mu \mathcal{B})^{\prime}-(\nu \mathbf{C B})^{\prime} \\
& =\mathbf{I} \mathbf{d}-\mathcal{B}^{\prime} \mu^{\prime}-\mathcal{B}^{\prime} \mathbf{C}^{\prime} \nu^{\prime}=\mathbf{I} \mathbf{d}-\mathcal{B}^{*} \bar{\mu}-\mathcal{B}^{*} \mathbf{C} \bar{\nu} \\
& =\mathcal{B}^{*}\left(\mathbf{I} \mathbf{d}-\bar{\mu} \mathcal{B}^{*}-\mathbf{C} \bar{\nu} \mathcal{B}^{*}\right) \mathcal{B}=\mathcal{B}^{*}\left(\mathbf{I} \mathbf{d}-\bar{\mu} \mathcal{B}^{*}-\nu \mathbf{C} \mathcal{B}^{*}\right) \mathcal{B},
\end{aligned}
$$

where we used the fact that $\mathcal{B}^{*} \mathcal{B}=\mathcal{B B}^{*}=\mathbf{I d}$. As a consequence, and using that both $\mathcal{B}$ and $\mathcal{B}^{*}$ are bijective in $L^{p}(\mathbf{C})$, we obtain that the bijectivity of the operator Id $-\mu \mathcal{B}-\nu \overline{\mathcal{B}}$ in $L_{\mathbf{R}}^{p}(\mathbf{C}) \oplus L_{\mathbf{R}}^{p}(\mathbf{C})$ is equivalent to that of $\mathbf{I d}-\bar{\mu} \mathcal{B}^{*}-\nu \mathbf{C B}^{*}$ in the dual space $L_{\mathbf{R}}^{p^{\prime}}(\mathbf{C}) \oplus L_{\mathbf{R}}^{p^{\prime}}(\mathbf{C})$. Similarly, one proves that

$$
\left(\mathbf{I d}-\mu \mathcal{B}^{*}-\nu \mathbf{C B}^{*}\right)^{\prime}=\mathcal{B}(\mathbf{I d}-\bar{\mu} \mathcal{B}-\nu \overline{\mathcal{B}}) \mathcal{B}^{*}
$$

Hence, the bijectivity of $\mathbf{I d}-\mu \mathcal{B}^{*}-\nu \mathbf{C B}^{*}$ in $L_{\mathbf{R}}^{p}(\mathbf{C}) \oplus L_{\mathbf{R}}^{p}(\mathbf{C})$ is equivalent to the bijectivity of $\mathbf{I d}-\bar{\mu} \mathcal{B}-\nu \overline{\mathcal{B}}$ in $L_{\mathbf{R}}^{p^{\prime}}(\mathbf{C}) \oplus L_{\mathbf{R}}^{p^{\prime}}(\mathbf{C})$.

Lemma 10. If $1<p<\infty, \omega \in A_{p}, \mu, \nu \in V M O$ have compact support, and $\||\mu|+|\nu|\|_{\infty} \leq k<1$, then the operators

$$
\text { Id }-\mu \mathcal{B}-\nu \overline{\mathcal{B}} \text { and } \quad \text { Id }-\mu \mathcal{B}^{*}-\nu \overline{\mathcal{B}^{*}}
$$


are Fredholm operators in $L^{p}(\omega)$.

Proof. We will show the claim for the operator $\operatorname{Id}-\mu \mathcal{B}-\nu \overline{\mathcal{B}}$. For $\operatorname{Id}-\mu \mathcal{B}^{*}-\nu \overline{\mathcal{B}^{*}}$ the proof follows similarly. It will be more convenient for us to write $\overline{\mathcal{B}}=\mathbf{C B}$. As in the proof of Theorem 1, we set

$$
P_{N}=\sum_{j=0}^{N}(\mu \mathcal{B}+\nu \mathbf{C B})^{j} .
$$

Then

$$
\begin{aligned}
& (\mathbf{I d}-\mu \mathcal{B}-\nu \mathbf{C B}) \circ P_{N-1}=\mathbf{I d}-(\mu \mathcal{B}+\nu \mathbf{C B})^{N}, \\
& P_{N-1} \circ(\mathbf{I d}-\mu \mathcal{B}+\nu \mathbf{C B})=\mathbf{I d}-(\mu \mathcal{B}+\nu \mathbf{C B})^{N} .
\end{aligned}
$$

We will show that

$$
(\mu \mathcal{B}+\nu \mathbf{C B})^{N}=R_{N}+K_{N}
$$

where $K_{N}$ is a compact operator, and $R_{N}$ is a bounded, linear operator such that

$$
\left\|R_{N} f\right\|_{L^{p}(\omega)} \leq C k^{N} N^{3}\|f\|_{L^{p}(\omega)} .
$$

Then, the Fredholm property follows immediately. To prove (20), let us write, for any two operators $T_{1}, T_{2}$,

$$
\left(T_{1}+T_{2}\right)^{N}=\sum_{\sigma \in\{1,2\}^{N}} T_{\sigma}
$$

where $\sigma \in\{1,2\}^{N}$ means that $\sigma=(\sigma(1), \ldots, \sigma(N))$ and $\sigma(j) \in\{1,2\}$ for all $j=$ $1, \ldots, N$, and

$$
T_{\sigma}=T_{\sigma(1)} T_{\sigma(2)} \ldots T_{\sigma(N)} .
$$

By choosing $T_{1}=\mu \mathcal{B}$ and $T_{2}=\nu \mathbf{C B}$, one sees that every $T_{\sigma(j)}$ can be written as

$$
T_{\sigma(j)}=M_{\sigma(j)} C_{\sigma(j)} \mathcal{B}
$$

being $M_{1}=\mu, M_{2}=\nu, C_{1}=\mathbf{I d}$ and $C_{2}=\mathbf{C}$. Thus

$$
T_{\sigma}=M_{\sigma(1)} C_{\sigma(1)} \mathcal{B} M_{\sigma(2)} C_{\sigma(2)} \mathcal{B} \ldots M_{\sigma(N)} C_{\sigma(N)} \mathcal{B} .
$$

Our main task consists of rewriting $T_{\sigma}$ as

$$
T_{\sigma}=M_{\sigma(1)} C_{\sigma(1)} M_{\sigma(2)} C_{\sigma(2)} \ldots M_{\sigma(N)} C_{\sigma(N)} B_{\sigma}+K_{\sigma} .
$$

for some compact operator $K_{\sigma}$ and some bounded operator $B_{\sigma} \in\left\{\mathcal{B}, \mathcal{B}^{*}\right\}^{N}$. If this is possible, then one gets that

$$
\begin{aligned}
\left(T_{1}+T_{2}\right)^{N} & =\sum_{\sigma \in\{1,2\}^{N}} M_{\sigma(1)} C_{\sigma(1)} M_{\sigma(2)} C_{\sigma(2)} \ldots M_{\sigma(N)} C_{\sigma(N)} B_{\sigma}+\sum_{\sigma \in\{1,2\}^{N}} K_{\sigma} \\
& =R_{N}+K_{N} .
\end{aligned}
$$

It is clear that $K_{N}$ is compact (it is a finite sum of compact operators). Moreover, from $B_{\sigma} \in\left\{\mathcal{B}, \mathcal{B}^{*}\right\}^{N}$, one has

$$
\left|B_{\sigma} f(z)\right| \leq \sum_{j=1}^{N}\left|\mathcal{B}^{n} f(z)\right|+\sum_{j=1}^{N}\left|\left(\mathcal{B}^{*}\right)^{n} f(z)\right| .
$$


Thus

$$
\begin{aligned}
\left|R_{N} f(z)\right| & \leq \sum_{\sigma \in\{1,2\}^{N}}\left|M_{\sigma(1)} C_{\sigma(1)} \ldots M_{\sigma(N)} C_{\sigma(N)} B_{\sigma} f(z)\right| \\
& \leq \sum_{\sigma \in\{1,2\}^{N}}\left|M_{\sigma(1)}(z)\right| \ldots\left|M_{\sigma(N)}(z)\right|\left(\sum_{n=1}^{N}\left|\mathcal{B}^{n} f(z)\right|+\sum_{j=1}^{N}\left|\left(\mathcal{B}^{*}\right)^{n} f(z)\right|\right) \\
& =\left(\sum_{n=1}^{N}\left|\mathcal{B}^{n} f(z)\right|+\sum_{j=1}^{N}\left|\left(\mathcal{B}^{*}\right)^{n} f(z)\right|\right) \cdot\left(\left|M_{1}(z)\right|+\left|M_{2}(z)\right|\right)^{N}
\end{aligned}
$$

Now, since $\left\|\mathcal{B}^{j} f\right\|_{L^{p}(\omega)} \leq C_{\omega} j^{2}\|f\|_{L^{p}(\omega)}$ (and similarly for $\left(\mathcal{B}^{*}\right)^{n}$, see (17)), one gets that

$$
\left\|R_{N} f\right\|_{L^{p}(\omega)} \leq\left\|\left|M_{1}\right|+\left|M_{2}\right|\right\|_{\infty}^{N} C_{\omega}\left(\sum_{j=1}^{N} j^{2}\right)\|f\|_{L^{p}(\omega)}=C k^{N} N^{3}\|f\|_{L^{p}(\omega)}
$$

and so (20) follows from the representation (21). To prove that representation (21) can be found, we need the help of Theorem 2, according to which the differences $K_{j}=\mathcal{B} M_{\sigma(j)}-M_{\sigma(j)} \mathcal{B}$ are compact. Thus,

$$
\begin{aligned}
T_{\sigma} & =M_{\sigma(1)} C_{\sigma(1)} \mathcal{B} M_{\sigma(2)} C_{\sigma(2)} \mathcal{B} \ldots M_{\sigma(N)} C_{\sigma(N)} \mathcal{B} \\
& =M_{\sigma(1)} C_{\sigma(1)} M_{\sigma(2)} \mathcal{B} C_{\sigma(2)} M_{\sigma(3)} \ldots \mathcal{B} C_{\sigma(N)} \mathcal{B}+K_{\sigma}
\end{aligned}
$$

where all the factors containning $K_{j}$ are includded in $K_{\sigma}$. In particular, $K_{\sigma}$ is compact. Now, by reminding that

$$
\mathrm{C} \mathcal{B}=\mathcal{B}^{*} \mathbf{C}
$$

we have that $\mathcal{B} C_{\sigma(j+1)}=C_{\sigma(j+1)} B_{j}$ for some $B_{j} \in\left\{\mathcal{B}, \mathcal{B}^{*}\right\}$. Thus

$$
T_{\sigma}=M_{\sigma(1)} C_{\sigma(1)} M_{\sigma(2)} C_{\sigma(2)} B_{1} M_{\sigma(3)} \ldots C_{\sigma(N)} B_{N-1} \mathcal{B}+K_{\sigma}
$$

Now, one can start again. On one hand, the differences $B_{j} M_{\sigma(j+2)}-M_{\sigma(j+2)} B_{j}$ are again compact, because $B_{j} \in\left\{\mathcal{B}, \mathcal{B}^{*}\right\}$ and $M_{\sigma(j+2)} \in V M O$. Moreover, the composition $B_{j} C_{\sigma(j+2)}$ can be writen as $C_{\sigma(j+2)} \tilde{B}_{j}$, where $\tilde{B}_{j}$ need not be the same as $B_{j}$ but still $\tilde{B}_{j} \in\left\{\mathcal{B}, \mathcal{B}^{*}\right\}$. So, with a little abbuse of notation, and after repeating this algorythm a total of $N-1$ times, one obtains (21). The claim follows.

Proof of Theorem 8. The equation we want to solve can be rewritten, at least formally, in the following terms

$$
(\mathbf{I} \mathbf{d}-\mu \mathcal{B}-\nu \overline{\mathcal{B}})(\bar{\partial} f)=g,
$$

so that we need to understand the $\mathbf{R}$-linear operator $T=\mathbf{I d}-\mu \mathcal{B}-\nu \overline{\mathcal{B}}$. By Lemma 10 , we know that $T$ is a Fredholm operator in $L^{p}(\omega), 1<p<\infty$. Now, we prove that it is also injective. Indeed, if

$$
T(h)=0
$$

for some $h \in L^{p}(\omega)$ and $\omega \in A_{p}$, it then follows that

$$
h=\mu \mathcal{B}(h)+\nu \overline{\mathcal{B}}(h)
$$

so that $h$ has compact support, and thus $h \in L^{1+\epsilon}(\mathbf{C})$ for some $\epsilon>0$ (arguing as in (18)). We are then reduced to show that

$$
T: L^{1+\epsilon}(\mathbf{C}) \rightarrow L^{1+\epsilon}(\mathbf{C}) \quad \text { is injective. }
$$


Let us first see how the proof finishes. Injectivity of $T$ in $L^{1+\epsilon}(\mathbf{C})$ gives us that $h=0$. Therefore, $T$ is injective also in $L^{p}(\omega)$. Being as well Fredholm, it is also surjective, so by the open map Theorem it has a bounded inverse $T^{-1}: L^{p}(\omega) \rightarrow L^{p}(\omega)$. As a consequence, given any $g \in L^{p}(\omega)$, the function

$$
f=\mathcal{C} T^{-1}(g)
$$

is well defined, and has derivatives in $L^{p}(\omega)$ satisfying the estimate

$$
\begin{aligned}
\|D f\|_{L^{p}(\omega)} & \leq\|\partial f\|_{L^{p}(\omega)}+\|\bar{\partial} f\|_{L^{p}(\omega)}=\left\|\mathcal{B} T^{-1}(g)\right\|_{L^{p}(\omega)}+\left\|T^{-1}(g)\right\|_{L^{p}(\omega)} \\
& \leq(C+1)\left\|T^{-1}(g)\right\|_{L^{p}(\omega)} \leq C\|g\|_{L^{p}(\omega)},
\end{aligned}
$$

because $\omega \in A_{p}$. Moreover, we see that $f$ solves the inhomogeneous equation

$$
\bar{\partial} f(z)-\mu(z) \partial f(z)-\nu(z) \overline{\partial f(z)}=g(z) .
$$

Finally, if there were two such solutions $f_{1}, f_{2}$, then their difference $F=f_{1}-f_{2}$ solves the homogeneous equation, and also $D F \in L^{p}(\omega)$. Thus

$$
T(\bar{\partial} F)=0 \text {. }
$$

By the injectivity of $T$ we get that $\bar{\partial} F=0$, and from $D F \in L^{p}(\omega)$ we get that $\partial F=0$, whence $F$ must be a constant.

We now prove the injectivity of $T$ in $L^{p}(\mathbf{C}), 1<p<\infty$. First, if $p \geq 2$ and $h \in L^{p}(\mathbf{C})$ is such that $T(h)=0$, then $h$ has compact support, whence $h \in L^{2}(\mathbf{C})$. But $\mathcal{B}, \overline{\mathcal{B}}$ are isometries in $L^{2}(\mathbf{C})$, whence

$$
\|h\|_{2} \leq k\|\mathcal{B} h\|_{2}=k\|f\|_{2}
$$

and thus $h=0$, as desired. For $p<2$, we recall from Lemma 9 that the bijectivity of $T$ in $L^{p}(\mathbf{C})$ is equivalent to that of $T^{\prime}=\mathbf{I d}-\bar{\mu} \mathcal{B}^{*}-\nu \overline{\mathcal{B}^{*}}$ in the dual space $L^{p}(\mathbf{C})$. For this, note that the injectivity of $T^{\prime}$ in $L^{p^{\prime}}(\mathbf{C})$ follows as above (since $p^{\prime} \geq 2$ ). Note also that, by Lemma 10 we know that $T^{\prime}$ is a Fredholm operator in $L^{p^{\prime}}(\mathbf{C})$, since $\bar{\mu}$ and $\nu$ are compactly supported $V M O$ functions. The claim follows.

\section{Applications}

We start this section by recalling that if $\mu, \nu \in L^{\infty}(\mathbf{C})$ are compactly supported with $\||\mu|+|\nu|\|_{\infty} \leq k<1$ then the equation

$$
\bar{\partial} \phi(z)-\mu(z) \partial \phi(z)-\nu(z) \overline{\partial \phi(z)}=0
$$

admits a unique homeomorphic $W_{\text {loc }}^{1,2}(\mathbf{C})$ solution $\phi: \mathbf{C} \rightarrow \mathbf{C}$ such that $|\phi(z)-z| \rightarrow 0$ as $|z| \rightarrow \infty$. We call it the principal solution, and it defines a global $K$-quasiconformal map, $K=\frac{1+k}{1-k}$. See the monograph [1].

Applications of Theorem 1 are based in the following change of variables lemma, which is already proved in [3, Lemma 14]. We rewrite it here for completeness.

Lemma 11. Given a compactly supported function $\mu \in L^{\infty}(\mathbf{C})$ such that $\|\mu\|_{\infty} \leq$ $k<1$, let $\phi$ denote the principal solution to the equation

$$
\bar{\partial} \phi(z)-\mu(z) \partial \phi(z)=0 .
$$

For a fixed weight $\omega$, let us define

$$
\eta(\zeta)=\omega\left(\phi^{-1}(\zeta)\right) J\left(\zeta, \phi^{-1}\right)^{1-\frac{p}{2}} .
$$

The following statements are equivalent: 
(a) For every $h \in L^{p}(\omega)$, the inhomogeneous equation

$$
\bar{\partial} f(z)-\mu(z) \partial f(z)=h(z)
$$

has a solution $f$ with $D f \in L^{p}(\omega)$ and

$$
\|D f\|_{L^{p}(\omega)} \leq C_{1}\|h\|_{L^{p}(\omega)} .
$$

(b) For every $\tilde{h} \in L^{p}(\eta)$, the equation

$$
\bar{\partial} g(\zeta)=\tilde{h}(\zeta)
$$

has a solution $g$ with $D g \in L^{p}(\eta)$ and

$$
\|D g\|_{L^{p}(\eta)} \leq C_{2}\|\tilde{h}\|_{L^{p}(\eta)} .
$$

Proof. Let us first assume that (b) holds. To get (a), we have to find a solution $f$ of (22) such that $D f \in L^{p}(\omega)$ with the estimate (23). To this end, we make in (22) the change of coordinates $g=f \circ \phi^{-1}$. We obtain for $g$ the following equation

$$
\bar{\partial} g(\zeta)=\tilde{h}(\zeta)
$$

where $\zeta=\phi(z)$ and

$$
\tilde{h}(\zeta)=h(z) \frac{\partial \phi(z)}{J(z, \phi)} .
$$

In order to apply the assumption (b), we must check that $\tilde{h} \in L^{p}(\eta)$. However,

$$
\begin{aligned}
\|\tilde{h}\|_{L^{p}(\eta)}^{p} & =\int|\tilde{h}(\zeta)|^{p} \eta(\zeta) \mathrm{d} \zeta=\int|\tilde{h}(\phi(z))|^{p} \omega(z) J(z, \phi)^{\frac{p}{2}} \mathrm{~d} z \\
& =\int|h(z)|^{p} \frac{\omega(z)}{\left(1-|\mu(z)|^{2}\right)^{\frac{p}{2}}} \mathrm{~d} z \leq \frac{1}{\left(1-k^{2}\right)^{\frac{p}{2}}}\|h\|_{L^{p}(\omega)}^{p} .
\end{aligned}
$$

Since $\tilde{h} \in L^{p}(\eta)$, (b) applies, and a solution $g$ to (26) can be found with the estimate

$$
\|D g\|_{L^{p}(\eta)} \leq C_{2}\|\tilde{h}\|_{L^{p}(\eta)} \leq \frac{C_{2}}{\left(1-k^{2}\right)^{\frac{1}{2}}}\|h\|_{L^{p}(\omega)} .
$$

With such a $g$, the function $f=g \circ \phi$ is well defined, and

$$
\begin{aligned}
\int|D f(z)|^{p} \omega(z) \mathrm{d} z & =\int|D g(\phi(z)) D \phi(z)|^{p} \omega(z) \mathrm{d} z \\
& =\int\left|D g(\zeta) D \phi\left(\phi^{-1}(\zeta)\right)\right|^{p} \omega\left(\phi^{-1}(\zeta)\right) J\left(\zeta, \phi^{-1}\right) \mathrm{d} \zeta \\
& \leq\left(\frac{1+k}{1-k}\right)^{\frac{p}{2}} \int|D g(\zeta)|^{p} J\left(\phi^{-1}(\zeta), \phi\right)^{\frac{p}{2}} \omega\left(\phi^{-1}(\zeta)\right) J\left(\zeta, \phi^{-1}\right) \mathrm{d} \zeta \\
& =\left(\frac{1+k}{1-k}\right)^{\frac{p}{2}} \int|D g(\zeta)|^{p} \eta(\zeta) \mathrm{d} \zeta .
\end{aligned}
$$

due to the $\frac{1+k}{1-k}$-quasiconformality of $\phi$. Moreover, $f$ satisfies the desired equation, and so (a) follows, with constant $C_{1}=\frac{C_{2}}{1-k}$.

To show that (a) implies (b), for a given $\tilde{h} \in L^{p}(\eta)$ we have to find a solution of (24) satisfying the estimate (25). Since this is a $\bar{\partial}$-equation, this could be done by simply convolving $\tilde{h}$ with the Cauchy kernel $\frac{1}{\pi z}$, but then the desired estimate for the solution $g$ cannot be obtained in this way, because at this point the weight $\eta$ is 
not known to belong to $A_{p}$. So we will proceed in a different maner. Namely, let $\tilde{h} \in L^{p}(\eta)$ be fixed, and set $h(z)=\tilde{h}(\phi(z)) \overline{\partial \phi(z)}\left(1-|\mu(z)|^{2}\right)$. Then

$$
\int|h(z)|^{p} \omega(z) \mathrm{d} z=\int|\tilde{h}(\zeta)|^{p}\left(1-\left|\mu\left(\phi^{-1}(\zeta)\right)\right|^{2}\right)^{p / 2} \eta(\zeta) \mathrm{d} \zeta \leq \int|\tilde{h}(\zeta)|^{p} \eta(\zeta) \mathrm{d} \zeta,
$$

and so $h \in L^{p}(\omega)$. By (a), the equation

$$
\bar{\partial} f(z)-\mu(z) \partial f(z)=h(z)
$$

has a unique solution $f$ with $D f \in L^{p}(\omega)$, and furthermore $\|D f\|_{L^{p}(\omega)} \leq C_{1}\|\tilde{h}\|_{L^{p}(\eta)}$. Now we simply set $g=f \circ \phi^{-1}$. By the chain rule, one gets that $\bar{\partial} g=\tilde{h}$, and

$$
\begin{aligned}
\int|D g(\zeta)|^{p} \eta(\zeta) \mathrm{d} \zeta & =\int\left|D g\left(\phi^{-1}(z)\right)\right|^{p} J\left(z, \phi^{-1}\right) \eta\left(\phi^{-1}(z)\right) \mathrm{d} z \\
& =\int\left|D\left(g \circ \phi^{-1}\right)(z)\left(D \phi^{-1}(z)\right)^{-1}\right|^{p} J\left(z, \phi^{-1}\right) \eta\left(\phi^{-1}(z)\right) \mathrm{d} z \\
& \leq \int|D f(z)|^{p}\left|D \phi\left(\phi^{-1}(z)\right)\right|^{p} J\left(z, \phi^{-1}\right) \eta\left(\phi^{-1}(z)\right) \mathrm{d} z \\
& \left.\leq\left(\frac{1+k}{1-k}\right)^{\frac{p}{2}} \int \mid D f(z)\right)\left.\right|^{p} J\left(\phi^{-1}(z), \phi\right)^{\frac{p}{2}} J\left(z, \phi^{-1}\right) \eta\left(\phi^{-1}(z)\right) \mathrm{d} z \\
& \left.=\left(\frac{1+k}{1-k}\right)^{\frac{p}{2}} \int \mid D f(z)\right)\left.\right|^{p} \omega(z) \mathrm{d} z .
\end{aligned}
$$

Thus, $\|D g\|_{L^{p}(\eta)} \leq C_{2}\|\tilde{h}\|_{L^{p}(\eta)}$ with $C_{2}=\left(\frac{1+k}{1-k}\right)^{\frac{1}{2}} C_{1}$, and (b) follows.

According to the previous Lemma, a priori estimates for $\bar{\partial}-\mu \partial$ in $L^{p}(\omega)$ are equivalent to a priori estimates for $\bar{\partial}$ in $L^{p}(\eta)$. However, by Theorem 1 , if $\omega$ is taken in $A_{p}$, the first statement holds, at least, when $\mu$ is compactly supported and belongs to $V M O$. We then obtain the following consequence.

Corollary 12. Let $\mu \in V M O$ be compactly supported, such that $\|\mu\|_{\infty}<1$, and let $\phi$ be the principal solution of

$$
\bar{\partial} \phi(z)-\mu(z) \partial \phi(z)=0 .
$$

If $1<p<\infty$ and $\omega \in A_{p}$, then the weight

$$
\eta(z)=\omega\left(\phi^{-1}(z)\right) J\left(z, \phi^{-1}\right)^{1-p / 2}
$$

belongs to $A_{p}$. Moreover, its $A_{p}$ constant $[\eta]_{A_{p}}$ can be bounded in terms of $\mu, p$ and $[\omega]_{A_{p}}$.

Proof. Under the above assumptions, by Theorem 1, we know that if $h \in L^{p}(\omega)$ then the equation $\bar{\partial} f-\mu \partial f=h$ can be found a solution $f$ with $D f \in L^{p}(\omega)$ and such that $\|D f\|_{L^{p}(\omega)} \leq C_{0}\|h\|_{L^{p}(\omega)}$, for some constant $C_{0}>0$ depending on $k, p$ and $[\omega]_{A_{p}}$. Equivalently, by Lemma 11, for every $\tilde{h} \in L^{p}(\eta)$ we can find a solution $g$ of the inhomogeneous Cauchy-Riemann equation

$$
\bar{\partial} g=\tilde{h},
$$

with $D g \in L^{p}(\eta)$ and in such a way that the estimate

$$
\|D g\|_{L^{p}(\eta)} \leq C\|\tilde{h}\|_{L^{p}(\eta)}
$$


holds for some constant $C$ depending on $C_{0}, k$ and $p$. Now, let us choose $\varphi \in \mathcal{C}_{0}^{\infty}(\mathbf{C})$ and set $\tilde{h}=\bar{\partial} \varphi$. Then of course $g=\varphi$ and $\partial \varphi=\mathcal{B}(\bar{\partial} \varphi)$, and the above inequality says that

whence the estimate

$$
\||\partial \varphi|+|\bar{\partial} \varphi|\|_{L^{p}(\eta)} \leq C\|\bar{\partial} \varphi\|_{L^{p}(\eta)},
$$

$$
\|\mathcal{B}(\psi)\|_{L^{p}(\eta)} \leq\left(C^{p}-1\right)^{\frac{1}{p}}\|\psi\|_{L^{p}(\eta)}
$$

holds for any $\psi \in \mathcal{D}^{*}=\left\{\psi \in \mathcal{C}_{c}^{\infty}(\mathbf{C}): \int \psi=0\right\}$. It turns out that $\mathcal{D}^{*}$ is a dense subclass of $L^{p}(\eta)$, provided that $\eta \in L_{\text {loc }}^{1}$ is a positive function with infinite mass. But this is actually the case. Indeed, one has

$$
\int_{D(0, R)} \eta(\zeta) \mathrm{d} \zeta=\int_{\phi^{-1}(D(0, R))} \omega(z) J(z, \phi)^{\frac{p}{2}} \mathrm{~d} z .
$$

Above, the integral on the right hand side certainly grows to infinite as $R \rightarrow \infty$. Otherwise, one would have that $J(\cdot, \phi)^{\frac{1}{2}} \in L^{p}(\omega)$. But $\phi$ is a principal quasiconformal map, hence $J(z, \phi)=1+O\left(1 /|z|^{2}\right)$ as $|z| \rightarrow \infty$. Thus for large enough $N>M>0$,

$$
\int_{M<|z|<N} J(z, \phi)^{\frac{p}{2}} \omega(z) \mathrm{d} z \geq C \int_{M<|z|<N} \omega(z) \mathrm{d} z
$$

and the last integral above blows up as $N \rightarrow \infty$, because $\omega$ is an $A_{p}$ weight.

Therefore, the estimate (27) holds for all $\psi$ in $L^{p}(\eta)$. By [17, Ch. V, Proposition $7]$, this implies that $\eta \in A_{p}$, and moreover, $[\eta]_{A_{p}}$ depends only on the constant $\left(C^{p}-1\right)^{\frac{1}{p}}$, that is, on $k, p$ and $[\omega]_{A_{p}}$.

The above Corollary is especially interesting in two particular cases. First, for the constant weight $\omega=1$ the above result says that

$$
J\left(\cdot, \phi^{-1}\right)^{1-p / 2} \in A_{p}, \quad 1<p<\infty .
$$

Without the $V M O$ assumption, this is only true for the smaller range $1+\|\mu\|_{\infty}<$ $p<1+\frac{1}{\|\mu\|_{\infty}}$ (see e.g. [2, Theorem 13.4.2]). Secondly, by setting $p=2$ in Corollary 12 we get the following.

Corollary 13. Let $\mu \in V M O$ be compactly supported, and assume that $\|\mu\|_{\infty}<$ 1. Let $\phi$ be the principal solution of

$$
\bar{\partial} \phi(z)-\mu(z) \partial \phi(z)=0
$$

Then, for every $\omega \in A_{2}$ one has $\omega \circ \phi^{-1} \in A_{2}$.

The above result drives us to the problem of finding what homeomorphisms $\phi$ preserve the $A_{p}$ classes under composition with $\phi^{-1}$. Note that preserving $A_{p}$ forces also the preservation of the space $B M O$ of functions with bounded mean oscillation, and thus such homeomorphisms $\phi$ must be quasiconformal [14]. However, at level of Muckenhoupt weights, the question is deeper. As an example, simply consider the weight

$$
\omega(z)=\frac{1}{|z|^{\alpha}}
$$

and its composition with the inverse of a radial stretching $\phi(z)=z|z|^{K-1}$. It is clear that the values of $p$ for which $A_{p}$ contains $\omega$ and $\omega \circ \phi^{-1}$ are not the same, whence preservation of $A_{p}$ requires something else. This question was solved by Johnson and Neugebauer [10] as follows. 
Theorem 14. Let $\phi: \mathbf{C} \rightarrow \mathbf{C}$ be $K$-quasiconformal. Then, the following statements are equivalent:

(1) If $\omega \in A_{2}$ then $\omega \circ \phi^{-1} \in A_{2}$ quantitatively.

(2) For a fixed $p \in(1, \infty)$, if $\omega \in A_{p}$ then $\omega \circ \phi^{-1} \in A_{p}$ quantitatively.

(3) $J\left(\cdot, \phi^{-1}\right) \in A_{p}$ for every $p \in(1, \infty)$.

It follows from Corollary 13 and Theorem 14 that, if $\mu \in V M O$ is compactly supported, $\|\mu\|_{\infty} \leq k<1$ and $\phi$ is the principal solution to the $\mathbf{C}$-linear equation $\bar{\partial} \phi=\mu \partial \phi$, then

$$
J\left(\cdot, \phi^{-1}\right) \in A_{p}, \quad \text { for every } p>1 .
$$

By quasisymmetry, the $A_{p}$ condition (5) for $J\left(\cdot, \phi^{-1}\right)$ also holds if $D$ is quasidisk. But then, after a change of coordinates, one gets for any disk $D^{\prime}$ and $D=\phi\left(D^{\prime}\right)$ that

$$
\left(f_{D} J\left(\cdot, \phi^{-1}\right)\right)\left(f_{D} J\left(\cdot, \phi^{-1}\right)^{1-p^{\prime}}\right)^{p-1}=\left(\left(f_{D^{\prime}} J(\cdot, \phi)\right)^{-1}\left(f_{D^{\prime}} J(\cdot, \phi)^{p^{\prime}}\right)^{\frac{1}{p^{\prime}}}\right)^{p},
$$

where $p^{\prime}=\frac{p}{p-1}$. As a consequence, we get that $J(\cdot, \phi)$ satisfies the reverse Hölder estimate (4) for any $1<p^{\prime}<\infty$. This shows Corollary 4.

It is not clear to the authors what is the role of $\mathbf{C}$-linearity in the above results. In other words, there seems to be no reason for Theorem 13 to fail if one replaces the C-linear equation by the generalized one, while mantainning the ellipticity, compact support and smoothness on the coefficients. Thus one may ask what is the class of weights $\omega>0$ for which the estimate

$$
\|D f\|_{L^{2}(\omega)} \leq C\|\bar{\partial} f-\mu \partial f-\nu \overline{\partial f}\|_{L^{2}(\omega)}
$$

holds for any $f \in \mathcal{C}_{0}^{\infty}(\mathbf{C})$. The following result, which is a counterpart of Lemma 11, explains this class contains $A_{p}$.

Lemma 15. To each pair $\mu, \nu \in L^{\infty}(\mathbf{C})$ of compactly supported functions with $\||\mu|+|\nu|\|_{\infty} \leq k<1$, let us associate, on one hand, the principal solution $\phi$ to the equation

$$
\bar{\partial} \phi(z)-\mu(z) \partial \phi(z)-\nu(z) \overline{\partial \phi(z)}=0
$$

and on the other, the function $\lambda$ defined by $\lambda \circ \phi=\frac{-2 i \nu}{1-|\mu|^{2}+|\nu|^{2}}$. For a fixed weight $\omega$, let us define

$$
\eta(\zeta)=\omega\left(\phi^{-1}(\zeta)\right) J\left(\zeta, \phi^{-1}\right)^{1-\frac{p}{2}}
$$

The following statements are equivalent:

(a) For every $h \in L^{p}(\omega)$, the equation

$$
\bar{\partial} f(z)-\mu(z) \partial f(z)-\nu(z) \overline{\partial f(z)}=h(z)
$$

has a solution $f$ with $D f \in L^{p}(\omega)$ and $\|D f\|_{L^{p}(\omega)} \leq C\|h\|_{L^{p}(\omega)}$.

(b) For every $\tilde{h} \in L^{p}(\eta)$, the equation

$$
\bar{\partial} g(\zeta)-\lambda(\zeta) \operatorname{Im}(\partial g(\zeta))=\tilde{h}(\zeta)
$$

has a solution $g$ with $D g \in L^{p}(\eta)$ and $\|D g\|_{L^{p}(\eta)} \leq C\|\tilde{h}\|_{L^{p}(\eta)}$.

Although the proof requires quite tedious calculations, it follows the scheme of Lemma 11, and thus we omit it. From this Lemma, the following one is a natural question to ask. 
Question 16. Let $\omega \in L_{\mathrm{loc}}^{1}(\mathbf{C})$ be such that $\omega(z)>0$ almost everywhere, and let $\lambda \in L^{\infty}(\mathbf{C})$ be a compactly supported $V M O$ function, such that $\|\lambda\|_{\infty}<1$. If the estimate

$$
\|D f\|_{L^{p}(\omega)} \leq C\|\bar{\partial} f-\lambda \operatorname{Im}(\partial f)\|_{L^{p}(\omega)}
$$

holds for every $f \in \mathcal{C}_{0}^{\infty}$, is it true that $\omega \in A_{2}$ ?

What we actually want is to find planar, elliptic, first order differential operators, different from the $\bar{\partial}$, that can be used to characterize the Muckenhoupt classes $A_{p}$. In this direction, an affirmative answer tho Question 16 would allow us to characterize $A_{2}$ weights as follows: given $\mu, \nu \in V M O$ uniformly elliptic and compactly supported, a positive a.e. function $\omega \in L_{\text {loc }}^{1}$ is an $A_{2}$ weight if and only if there is a constant $C \geq 1$ such that

$$
\|D f\|_{L^{2}(\omega)} \leq C\|\bar{\partial} f-\mu \partial f-\nu \overline{\partial f}\|_{L^{2}(\omega)}, \quad \text { for every } f \in \mathcal{C}_{0}^{\infty}(\mathbf{C}) .
$$

Note that if one assumes $\||\mu|+|\nu|\|_{\infty}<\epsilon$ for some $\epsilon>0$, then (28) implies that

$$
\|\partial f\|_{L^{2}(\omega)}^{2}+\|\bar{\partial} f\|_{L^{2}(\omega)} \leq C\|\bar{\partial} f\|_{L^{2}(\omega)}+C \epsilon\|\partial f\|_{L^{2}(\omega)} .
$$

In particular, if for some reason $\epsilon<\frac{1}{C}$ then one gets

$$
\|\partial f\|_{L^{2}(\omega)} \leq \frac{C-1}{1-C \epsilon}\|\bar{\partial} f\|_{L^{2}(\omega)} .
$$

From the above estimate, weighted bounds for $\mathcal{B}$ easily follow, and so in this case such a characterization holds.

Acknowledgements. Sections 1 and 2 of the present paper were obtained by the second author in [4], jointly with J. Mateu and J. Orobitg. The authors wish to thank the referee for careful reading and comments. They were partially supported by projects 2009-SGR-420 (Generalitat de Catalunya), 2010-MTM-15657 (Spanish Ministry of Science), NF-129254 (Programa Ramón y Cajal) and UTMIX-PTC-036 and CONACYT (México).

\section{References}

[1] Astala, K., T. Iwaniec, and G. Martin: Pucci's conjecture and the Alexandrov inequality for elliptic PDEs in the plane. - J. Reine Angew. Math. 591, 2006, 49-74.

[2] Astala, K., T. Iwaniec, and G. Martin: Elliptic partial differential equations and quasiconformal mappings in the plane. - Princeton Math. Ser. 48, Princeton Univ. Press, Princeton, NJ, 2009.

[3] Astala, K., T. Iwaniec, and E. Saksman: Beltrami operators in the plane. - Duke Math. J. 107:1, 2001, 27-56.

[4] Cruz Barriguete, V. A.: Soluciones de la ecuación de Beltrami con coeficiente regular. Ph.D. Thesis, Departament de Matemàtiques, Universitat Autònoma de Barcelona, 2011.

[5] Cruz, V., J. Mateu, and J. Orobitg: Beltrami equation with coefficient in Sobolev and Besov spaces. - Preprint, 2011.

[6] Duonndikoetxea, J.: Fourier analysis. - Grad. Stud. Math. 29, Amer. Math. Soc., Providence, RI, 2001.

[7] García-Cuerva, J., and J. L. Rubio de Francia: Weighted norm inequalities and related topics. - North-Holland Math. Stud. 116, North-Holland Publ. Co., Amsterdam, 1985.

[8] Hanche-Olsen, H., and H. Holden: The Kolmogorov-Riesz compactness theorem. - Expo. Math. 28:4, 2010, 385-394. 
[9] IwANiEC, T.: $L^{p}$-theory of quasiregular mappings. - In: Quasiconformal space mappings, Lecture Notes in Math. 1508, Springer, Berlin, 1992, 39-64.

[10] Johnson, R., and C. J. Neugebauer: Homeomorphisms preserving $A_{p}$. - Rev. Mat. Iberoamericana 3:2, 1987, 249-273.

[11] Koski, A.: Singular integrals and Beltrami type operators in the plane and beyond. - Master Thesis, Department of Mathematics and Statistics, University of Helsinki, 2011.

[12] Krantz, S. G., and S.-Y. Li: Boundedness and compactness of integral operators on spaces of homogeneous type and applications. II. - J. Math. Anal. Appl. 258:2, 2001, 642-657.

[13] PÉREz, C.: Sharp estimates for commutators of singular integrals via iterations of the HardyLittlewood maximal function. - J. Fourier Anal. Appl. 3:6, 1997, 743-756.

[14] Reimann, H. M.: Functions of bounded mean oscillation and quasiconformal mappings. Comment. Math. Helv. 49, 1974, 260-276.

[15] Segovia, C., and J. L. Torrea: Weighted inequalities for commutators of fractional and singular integrals. - Publ. Mat. 35:1, 1991, 209-235.

[16] Stein, E. M.: Singular integrals and differentiability properties of functions. - Princeton Math. Ser. 30, Princeton Univ. Press, Princeton, NJ, 1970.

[17] Stein, E. M.: Harmonic analysis: real-variable methods, orthogonality, and oscillatory integrals. - Princeton Math. Ser. 43, Princeton Univ. Press, Princeton, NJ, 1993.

[18] Uchiyama, A.: On the compactness of operators of Hankel type. - Tôhoku Math. J. (2) 30:1, $1978,163-171$.

Received 23 December 2011 • Revised received 9 October 2012 • Accepted 29 October 2012 